\title{
COMPLEX MAXWELL AND EINSTEIN FIELDS
}

\author{
EZRA T. NEWMAN* \\ University of Pittsburgh, U.S.A.
}

\begin{abstract}
We consider the class of regular (in a certain precise sense) null vector fields, $l_{\mu}$ which have the following properties; they are (1) tangent to geodesics, (2) diverging, (3) shear free, (4) twist (or curl) free. It is well known that the vacuum Einstein fields whose principle null vector field (pnvf) satisfies (1)-(4) are the Robinson-Trautman (1962) (RT) metrics and those which satisfy (1) $(3)$ are the algebraically special twisting metrics, (Kerr, 1963). To understand these metrics better we ask for those Maxwell fields (in flat space) whose pnvf also satisfy conditions (1)-(4) and (1)-(3). It can be shown that (1)-(4) imply (and are implied by) that the Maxwell field is a LienardWiechart (LW) field. (This establishes the analogy between the RT metrics and the LW fields.) Conditions (1) $-(3)$ imply that the Maxwell field is a complex LW field. (We mean by this that if the Maxwell equations are complexified (Newman, 1973) (in complex Minkowski space) then the real solution in question is induced from the complex solution which is associated with a charged particle moving along an arbitrary complex world line.) Finally it can be shown that the Einstein equations can be complexified and that the algebraically special twisting metrics can be interpreted as if they had a point source moving in the complex manifold and are thus analogous to the complex LW fields.
\end{abstract}

\section{References}

Kerr, R. P.: 1963, Phys. Rev. Letters 11, 237.

Newman, E. T.: 1973, J. Math. Phys. 14, 102.

Robinson, I. and Trautman, A.: 1962, Proc. Roy. Soc. A265, 462.

* This work was done in collaboration with $\mathrm{R}$. Lind. 